\title{
RETRACTED ARTICLE: Anticoagulant agents for the prevention of hemodialysis catheter-related complications: systematic review and meta-analysis of prospective randomized controlled trials
}

\author{
Jinrui Liu ${ }^{1}$ Chang'an Wang ${ }^{1} \cdot$ Hongfei Zhao ${ }^{2} \cdot J^{\prime n g h u a ~ Z h a n g ~}{ }^{1} \cdot \mathrm{Jie} \mathrm{Ma}^{4} \cdot$ \\ Yuanyuan $\mathrm{Hou}^{3} \cdot$ Hongbin $\mathrm{Zou}^{4}$
}

Received: 18 May 2015 / Accepted: 19 October 2015 / Published online: 27 November 2015

(C) Springer Science+Business Media Dordrecht 2015

This article was retracted by the first author due to duplicate publication. The Editor-in-Chief and Springer were informed by the author that a similar version of the article was previously published in the International Journal of Clinical and Experimental Medicine.

The online version of this article contains the full text of the retracted article as electronic supplementary material.

Electronic supplementary material The online version of this article (doi:10.1007/s11255-015-1143-5) contains supplementary material, which is available to authorized users.

Hongbin Zou

zhb4663615@163.com

1 Department of Renal Transplantation and Nephropathy, The Seventh People's Hospital of Zhengzhou City,

Zhengzhou 45000, Henan, China

2 Department of Blood Transfusion, The Seventh People's Hospital of Zhengzhou City, Zhengzhou 45000, Henan, China

3 Department of Pediatrics, The Second Part of the First Hospital, Jilin University, Changchun 130041, Jilin, China

4 Department of Nephropathy, The Second Hospital of Jilin University, Street No. 218, Changchun 130041, Jilin, China 